\title{
BMJ Open Efficacy of non-invasive ventilation and oxygen therapy on immunocompromised patients with acute hypoxaemic respiratory failure: protocol for a systematic review and meta-analysis of randomised controlled trials
}

Zongru Li, ${ }^{1}$ Tao Wang, ${ }^{1}$ Yi Yang, ${ }^{1}$ Lixi Zhang, ${ }^{2}$ Meng Wang, ${ }^{3}$ Gang Liu, ${ }^{4}$ Kun He, ${ }^{5}$ Juhong Shi, ${ }^{6}$ Jianqiang $\mathrm{He},{ }^{1}$ Yong Ma, ${ }^{1} \mathrm{Yi} \mathrm{Li},{ }^{1}$ Huadong $\mathrm{Zhu},{ }^{1}$ Xuezhong $\mathrm{Yu}^{1}$
To cite: Li Z, Wang T, Yang Y, et al. Efficacy of non-invasive ventilation and oxygen therapy on immunocompromised patients with acute hypoxaemic respiratory failure: protocol for a systematic review and meta-analysis of randomised controlled trials. BMJ Open 2017;7:e015335. doi:10.1136/ bmjopen-2016-015335

- Prepublication history and additional material for this paper are available online. To view these files please visit the journal online (http://dx.doi. org/10.1136/bmjopen-2016015335).

\section{ZL, TW and YY contributed} equally.

Received 29 November 2016 Revised 20 April 2017 Accepted 17 May 2017

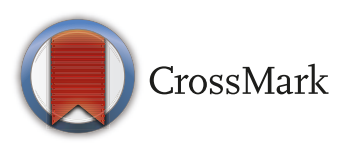

For numbered affiliations see end of article.

\section{Correspondence to} DrYi Li; billliyi@126.com and DrXuezhong Yu; yxz@medmail. com.cn

\begin{abstract}
Background The number of immunocompromised patients has increased in recent years. Acute respiratory failure is a common complication leading to intensive care unit (ICU) admission and high mortality among such patients. The use of non-invasive ventilation (NIV) or oxygen therapy among these patients remains controversial, according to the inconsistent results of several randomised clinical trials (RCTs). This meta-analysis aims to evaluate whether NIV or oxygen therapy is the more appropriate initial oxygenation strategy for the immunocompromised patients with acute respiratory failure.
\end{abstract}

Method We will search all the RCTs that compared the efficacy of NIV and oxygen therapy on immunocompromised adult patients with acute hypoxaemic respiratory failure on the major databases (Cochrane Library, MEDLINE, EMBASE, Web of Science and others), conference proceedings and grey literature. Eligible RCTs will be included in accordance with the pre-specified eligibility criteria. The risk of bias will be assessed using the Cochrane Collaboration criteria and the quality of evidence will be assessed with the Grading of Recommendations Assessment, Development and Evaluation system. Data will be extracted with a standardised form and analysed using RevMan V.5.3 analyses software. Heterogeneity will be assessed using $\mathrm{I}^{2}$ statistic and the source of which will be investigated. Publication bias will be identified with the funnel plot. Ethics and dissemination Ethical approval is not required since it is not carried out in humans. The systematic review will be published in peer-reviewed journals and disseminated extensively through conferences.

\section{BACKGROUND}

Description of the problem

Numerous factors such as the epidemic of AIDS, ${ }^{1}$ improved survival rates of active malignancies, ${ }^{23}$ innovative advances in organ

\section{Strengths and limitations of this study}

- There is no existing meta-analysis on the use of non-invasive ventilation (NIV) among immunocompromised patients with acute hypoxaemic respiratory failure.

- This meta-analysis includes only randomised clinical trials and will thus provide the highest quality of evidence for clinical practice.

- Subgroup analysis based on different levels of severity might support the use of NIV in more severe patients

The number of included studies is likely to be small.

transplantation, ${ }^{4}$ better outcomes of allogeneic hematopoietic cell transplantations ${ }^{5}$ and the more common application of immunosuppressive therapy have contributed to an increasing number of immunocompromised patients. These patients are more vulnerable to infection due to their inadequate immune response to foreign antigens. ${ }^{6}$ Some life-threatening complications can lead to requirement of intensive care unit (ICU) admission for these patients, among which acute respiratory failure (ARF) is the most common with particularly high mortality. ${ }^{7} \mathrm{ARF}$ is a relatively sudden onset of dysfunction of the respiratory system, and the most common causes among immunocompromised patients are immunosuppression-related infection, ${ }^{8-10}$ disease-specific infiltration, ${ }^{11}$ chemotherapy-associated organ toxicity ${ }^{12}$ and idiopathic pneumonia syndrome associated with graftversus-host disease (GVHD). ${ }^{13}$ For patients with severe ARF, invasive ventilation is 
required in order to support alveolar ventilation; however, such intervention also contributes to high mortality due to the risk of ventilator-associated pneumonia. ${ }^{14}$ Therefore, the strategy of delivering oxygen is of great importance for improvement of oxygenation, which may lead to reduction of intubation rate and mortality.

\section{Description of the intervention}

The percentage of usage of non-invasive ventilation (NIV) has increased from 29\% in 1997 to $42 \%$ in 2011 among patients with ARF. ${ }^{15}$ The benefits NIV may bring are associated not only with the degree of inspiratory workload spared by the positive airway pressure provided but also with the invasive-ventilation-associated complications that are prevented by NIV. ${ }^{16-18}$ However, the failure of NIV was identified as an independent risk factor for ICU mortality, which occurred in half of the critically ill haematological patients. ${ }^{19}$ Oxygen therapy, conducted via either nasal cannula, venturi mask or reservoir mask, is the basic technique used in patients with acute lung injury. Patients might benefit from oxygen therapy for less discomfort or intolerance compared with NIV. ${ }^{20}$

\section{Why is it important to do this review?}

The use of NIV was recommended for patients with acute hypoxaemic respiratory failure in the setting of immunosuppression weak (grade 2B), ${ }^{21}$ based on Antonelli et als and Hilbert et $a l$ s randomised clinical trials (RCTs) published in 2000 and 2001, ${ }^{22}{ }^{23}$ respectively. Findings of these two studies showed that NIV was associated with reduced intubation rate and mortality in immunocompromised patients with acute hypoxaemic respiratory failure. However, findings to the contrary can be found in the publications of Wermke $e t a l^{24}$ and Lemiale $e t a l^{25}$ Both of their studies showed that NIV was not associated with lowered intubation rate or mortality compared with oxygen therapy. No solid conclusion could be drawn based on the data currently available according to the reviews published in recent years, except that NIV should be applied with great caution in this group of patients. ${ }^{2627}$ Since the application of NIV in immunocompromised patients with acute hypoxaemic respiratory failure remains controversial, a systematic review and meta-analysis that summarises all the available RCTs is called for to provide guidance for the management of this group of patients. To our knowledge, no meta-analysis about this topic has yet been published.

The aim of this study is to determine the efficacy of NIV in comparison with oxygen therapy as the initial oxygenation strategy on the immunocompromised patients with acute hypoxaemic respiratory failure, with respect to mortality, intubation rate and hospital length of stay, and also to explore the patient selection strategy for the initial oxygenation strategy. Furthermore, the proposed systematic review will provide evidence for the use of NIV in subgroups of patients with different levels of disease severity, cause of immunosuppression, cause of ARF and others.

\section{METHOD}

This protocol of systematic review was reported following Preferred Reporting Items for Systematic Review and Meta-Analyses Protocol guidelines. ${ }^{28}$

Eligibility criteria: participants, interventions, comparisons and outcomes

Type of studies

Only RCTs will be included. Other types of studies such as observational studies, cohort studies, case-control studies and laboratory studies will be excluded. All included studies have to comply with international ethical rules.

\section{Type of participants}

This review will include RCTs involving immunocompromised adult patients with ARF. The immunocompromised patients include patients with haematological malignancy, solid cancer, AIDS or those receiving corticosteroid or cytotoxic therapy, or those having gone through solid organ or stem cell transplantation. Acute hypoxaemic respiratory failure is defined as respiratory rate $>30$ breaths $/ \mathrm{min}$, $\mathrm{PaO}_{2}<60 \mathrm{~mm} \mathrm{Hg}$ on room air or laboured breathing, and a partial pressure of arterial carbon dioxide not higher than $45 \mathrm{~mm} \mathrm{Hg} .{ }^{19}$ RCTs with a subgroup of participants who meet the criteria above will also be included, on the condition that the data of outcome for this subgroup are available. It should be noted that RCTs will be included if more than $85 \%$ of the involved participants meet the eligibility criteria, even if the outcomes of these eligible participants are unavailable.

\section{Type of intervention}

The intervention group refers to patients treated with NIV, which includes two main modes: continuous positive airway pressure (CPAP) and bi-level positive airway pressure (BiPAP).

The control group refers to patients treated with oxygen therapy. High-flow nasal oxygen (HFNO) therapy is a relatively new method of oxygen therapy that differentiates itself from oxygen therapy by providing positive pressure. ${ }^{29}$ Patients who have been treated with HFNO are therefore excluded from this study. As for the reports where mixed usage of HFNO and oxygen were adopted, the trial will be included if the data of sole oxygen therapy can be retrieved. We will include RCTs that directly compare NIV with oxygen therapy as the initial oxygenation strategy for acute respiratory failure, regardless of whether the other oxygenation method was applied later.

\section{Type of outcome measures}

- Primary outcome:

(1) Mortality: hospital mortality, ICU mortality and mortality at the last time available, in case that mortalities of all included studies were not measured at the same time period.

- Secondary outcome:

(1) Incidence of tracheal intubation.

(2) Length of ICU stay.

(3) Length of hospital stay. 
(4) Complications related to NIV.

(5) Rate of pulmonary complications not present on admission.

Eligible RCTs should include at least one of the primary outcomes listed above.

\section{Search strategy for identification of studies}

\section{Electronic searches}

Two reviewers (YY and LZ) will search the following databases: The Cochrane Library, MEDLINE, EMBASE, Web of Science, CINAHL, LILACS and PEDro by using database-specific search strategies. These electronic databases will be searched from January 1980 to date. No limitation of language or publication status will be applied. The filter for clinical trials will be used for each database. The following keywords will be used during the database searching: immunosuppression, hematological malignancy, cancer, transplantation, corticosteroid, cytotoxic, non-invasive ventilation and acute respiratory failure. The detailed search strategy can be found in the online supplementary 1 and 2.

\section{Searching other resources}

The references of relevant studies and review articles will be sought for potential information missing in database search. Conference proceedings and grey literature will be checked. The experts in the field will be contacted to identify published and unpublished trials. We will also access http:/ /www.clinicaltrials.gov and ClinicalTrials.gov for ongoing and unpublished studies, and the conductors or authors will be contacted for further information if necessary.

\section{Screening of studies}

All results identified by the search strategy will be screened by two reviewers (ZL and TW) independently. Initial screening will be performed on titles and abstracts, respectively, where irrelevant studies will be excluded according to the eligibility criteria; full texts of the remaining studies will subsequently be downloaded and screened. Reasons of exclusion will be documented and classified. Any disagreements between the reviewers will be solved through discussion, and the third author (YL) will be consulted if consensus cannot be reached.

\section{Data extraction and management}

Two reviewers (ZL and TW) will independently extract all the data in the included studies. A standard form will be used in extracting the following data:

1. Characteristics of the study: design, setting, method of randomisation, allocation concealment, blinding and dropouts.

2. Participants: number enrolled in each group, gender, age, respiratory rate, oxygen saturation, oxygenation index $\left(\mathrm{PaO}_{2} / \mathrm{FiO}_{2}\right)$, Sequential Organ Failure Assessment (SOFA), Acute Physiology and Chronic Health Evaluation (APACHE II), new Simplified
Acute Physiology Scale (SAPS II), cause of ARF and cause of immunosuppression.

3. Interventions: mode of NIV (CPAP or BiPAP), frequency and duration of ventilation; oxygen therapy and co-interventions.

4. Outcome: primary outcomes and secondary outcomes listed above.

Authors will be contacted for the missing data or subgroup data that are unavailable from the text. The consistency of data will be ensured by these two reviewers.

\section{Assessment of risk of bias}

For the included articles, the risk of bias will be assessed by two reviewers (YY and LZ) independently, using the Cochrane Collaboration criteria, ${ }^{30}$ which includes random sequence generation, allocation concealment, blinding of participants and outcome assessment, incomplete outcome data and selective outcome reporting. Each criterion will be explicitly judged and classified as 'low risk', 'high risk' or 'unclear risk'. Authors will be contacted for supplemental information if details for assessment reported in the text are considered inadequate. The risk will be rated as 'unclear' if no further information is obtained. The result of assessment of each study will be summarised in a chart. Overall risk of bias for each study will be defined as 'low' if risk of all bias components is ranked as 'low', 'moderate' if at least one component is ranked 'unclear' with no component ranked as 'high' or 'high' if one or more component is ranked as having a 'high' risk of bias.

\section{Data analyses and assessment of heterogeneity}

\section{Measures of treatment effect}

The statistical analyses will be performed using RevMan V.5.3 analyses software of the Cochrane Collaboration. Continuous data such as length of ICU stay and length of hospital stay will be presented as mean differences with $95 \%$ CIs. Dichotomous data such as the number of intubation and death will be presented as risk ratios with 95\% CIs. When the rates rather than the numbers are reported, we will calculate the numbers based on the data provided.

\section{Dealing with missing data}

Missing data will be dealt with following the recommendations of the Cochrane Handbook for Systematic Reviews of Interventions. Corresponding authors will be contacted for further information. If the missing data cannot be obtained, we will specify the assumptions of the methods used to cope with missing data according to the cause of data loss (ie, random dropout or poor outcome). We will perform sensitivity analyses to evaluate how sensitive results are to the changes in the assumptions that are made. In the Discussion section of the review, we will analyse the potential impact the missing data may have on the findings of the review. 
Assessment of heterogeneity

Before any outcome is pooled, we will assess the impact of heterogeneity using $\chi^{2}$ test and $\mathrm{I}^{2}$ statistic (classified as low $(<40 \%)$, moderate $(40 \%-60 \%)$ or high $(>60 \%))$. $\mathrm{I}^{2}$ values greater than $60 \%$ will be considered as having substantial heterogeneity. If substantial heterogeneity is present, we will investigate the potential source of heterogeneity by conducting exploratory analyses.

\section{Assessment of reporting biases}

Protocols of included trials will be searched using the databases mentioned above. We will contact the authors to obtain complete data of the protocols' envisioned outcomes as well as reasons for the non-reporting of certain outcomes. Publication bias will be assessed by visual analysis of the funnel plot if the number of included studies is equal to or greater than 10 .

\section{Subgroup analysis and investigation of heterogeneity}

Subgroup analysis will be used to explore potential sources of heterogeneity. Possible sources of heterogeneity are as follows:

1. Severity of acute respiratory failure before randomisation indicated by oxygenation index, SOFA, SAPS II and APACHE II as the baseline characteristics of included patients.

2. Different causes of immunosuppression, that is, HIV or non-HIV.

3. Different causes of acute respiratory failure.

4. Types of NIV (CPAP or BiPAP).

Sensitivity analysis will be carried out to assess the effect of exclusion of the studies with high overall risk of bias or the studies in which immunocompromised patients with ARF are a subgroup of the overall participants.

\section{Assessment of pooled effect estimates}

As to the pooled assessment of treatment effect, the Mantel-Haenszel method will be used for fixed-effects estimation and the DerSimonian and Laird method for random-effects estimation. The random-effects model was preferred if heterogeneity of treatment effects was present; otherwise, a fixed-effect model would be used. p Values $<0.05$ will be considered statistically significant. Results will be presented in tables and discussed afterwards where data aggregation is not possible due to substantial heterogeneity.

The quality of evidence contributing to pooled effect estimates will be evaluated following the principle of the Grading of Recommendations Assessment, Development and Evaluation (GRADE) system. ${ }^{31}$ According to the GRADE system, the quality of each evidence from RCT is considered to be high and will be downgraded with the presence of study limitations, imprecision, inconsistency, indirectness or publication bias.

Finally, all the findings will be summarised in a table following the GRADE principles.

\section{DISCUSSION}

The benefit of NIV among immunocompromised patients with acute hypoxaemic respiratory failure is unclear. The recommendation of the use of NIV in those patients has been challenged by the different results of the RCTs conducted in recent years. This systematic review and meta-analysis will synthesise evidences from all the available RCTs, which would be useful for clinicians regarding the use of NIV or oxygen therapy in those patients. Besides, subgroup analysis will be performed to find out more specific indications for clinical decision making.

Patients who have been treated with HFNO will not be included in our studies since HFNO is distinctively different from oxygen therapy in terms of equipment, cost and tolerance. HFNO requires more advanced equipment, thus it is not as popularised as standard oxygen therapy especially in developing countries such as China. Besides, the effect of HFNO is different from traditional oxygen therapy. Maggiore $e t a l$ s study showed that HFNO results in fewer oxygen desaturations, lower reintubation rate and less discomfort compared with oxygen therapy after extubation. ${ }^{32}$ Also, in Frat et als RCT conducted among patients with acute hypoxaemic respiratory failure, HFNO resulted in reduced mortality compared with standard oxygen therapy or NIV. ${ }^{33}$ Therefore, exclusion should be made so that HFNO would not become a confounding factor when we compare NIV with oxygen therapy.

HIV patients is a specific group and thus will be analysed in subgroup analysis. A systematic review conducted by our team showed that NIV had great advantage over invasive ventilation for HIV patients, and this advantage is less obvious among non-HIV patients. ${ }^{34}$ Furthermore, recent studies showed a higher mortality rate of Pneumocystis pneumonia infection in non-HIV patients in comparison with HIV patients. ${ }^{35}$ Therefore, we propose a hypothesis that the effect of NIV is different between HIV and non-HIV patients, which will be examined by subgroup analysis in this meta-analysis.

The overall purpose of this study is to determine whether NIV is better than oxygen therapy as the initial oxygenation strategy in adult immunocompromised patients with acute hypoxaemic respiratory failure. We will also explore the patient selection strategy for the initial oxygenation strategy with respect to severity, cause of immunosuppression and cause of ARF. The finding of this meta-analysis could also provide guidance for the RCTs in the future to find out the characteristics of patients who might benefit from NIV.

\section{Author affiliations}

${ }^{1}$ Emergency Department, Peking Union Medical College Hospital, Beijing, China ${ }^{2}$ Department of Cardiology, Peking Union Medical College Hospital, Beijing, China ${ }^{3}$ Department of Science and Technology, Beijing Tiantan Hospital, Capital Medical University, Beijing, China

${ }^{4}$ Department of Orthopaedics, Peking Union Medical College Hospital, Beijing, China ${ }^{5}$ Department of Gastroenterology, Peking Union Medical College Hospital, Beijing, China 
${ }^{6}$ Department of Pulmonary Medicine, Peking Union Medical College Hospital, Beijing, China

Contributors YL, TW and ZL developed the initial idea for this protocol. LZ, GL and $\mathrm{KH}$ contributed to the search strategy. Data abstraction forms were designed by MW. JS was consulted about intensive care and pulmonary medicine. JH, YM, $Y L, H Z$ and $X Y$ were consulted about emergency medicine. YY and ZL contributed to the original draft. YL, TW and LZ were responsible for the revision of the draft. $\mathrm{ZL}, \mathrm{TW}$ and $\mathrm{YY}$ were considered equal contributors to this article. All of the authors approved the final work prior to submission.

Funding National Natural Science Foundation of China No. 81550034 (YL). Competing interests None declared.

Ethics approval Ethics approval is not a requirement since no primary data will be collected from humans. This study is expected to provide evidence for the initial ventilation in immunocompromised patients with acute hypoxaemic respiratory failure. The finding of this study will be submitted to a peer-reviewed journal for publication and will be disseminated in conferences.

Provenance and peer review Not commissioned; externally peer reviewed.

Open Access This is an Open Access article distributed in accordance with the Creative Commons Attribution Non Commercial (CC BY-NC 4.0) license, which permits others to distribute, remix, adapt, build upon this work non-commercially, and license their derivative works on different terms, provided the original work is properly cited and the use is non-commercial. See: http://creativecommons.org/ licenses/by-nc/4.0/

(C) Article author(s) (or their employer(s) unless otherwise stated in the text of the article) 2017. All rights reserved. No commercial use is permitted unless otherwise expressly granted.

\section{REFERENCES}

1. Simon V, Ho DD, Abdool Karim Q. HIV/AIDS epidemiology, pathogenesis, prevention, and treatment. Lancet 2006;368:489-504.

2. Coleman MP, Quaresma M, Berrino F, et al. Cancer survival in five continents: a worldwide population-based study (CONCORD). Lancet Oncol 2008;9:730-56.

3. Fisher R, Dangoisse C, Crichton S, et al. Short-term and mediumterm survival of critically ill patients with solid tumours admitted to the intensive care unit: a retrospective analysis. BMJ Open 2016;6:e11363.

4. Salvadori M, Bertoni E. What's new in clinical solid organ transplantation by 2013. World J Transplant 2014;4:243-66.

5. Gooley TA, Chien JW, Pergam SA, et al. Reduced mortality after allogeneic hematopoietic-cell transplantation. N Engl J Med 2010;363:2091-101.

6. Chen XH, Yin YJ, Zhang JX. Sepsis and immune response. World J Emerg Med 2011;2:88-92.

7. Azoulay E, Mokart D, Pène F, et al. Outcomes of critically ill patients with hematologic malignancies: prospective multicenter data from France and Belgium - a groupe de recherche respiratoire en réanimation onco-hématologique study. J Clin Oncol 2013;31:2810-8.

8. Squadrone V, Ferreyra G, Ranieri VM. Non-invasive ventilation in patients with hematologic malignancy: a new prospective. Minerva Anestesiol 2015;81:1118-26.

9. Li Y, Ghannoum M, Deng C, et al. Pneumocystis pneumonia in patients with inflammatory or autoimmune diseases: usefulness of lymphocyte subtyping. Int $J$ Infect Dis 2017;57:108-15.

10. Ganzel C, Becker J, Mintz PD, et al. Hyperleukocytosis, leukostasis and leukapheresis: practice management. Blood Rev 2012;26:117-22.

11. Bhalla KS, Wilczynski SW, Abushamaa AM, et al. Pulmonary toxicity of induction chemotherapy prior to standard or high-dose chemotherapy with autologous hematopoietic support. Am J Respir Crit Care Med 2000;161:17-25.

12. Zhu KE, Hu JY, Zhang T, et al. Incidence, risks, and outcome of idiopathic pneumonia syndrome early after allogeneic hematopoietic stem cell transplantation. Eur J Haematol 2008;81:461-6.
13. Mehta A, Bhagat R. Preventing ventilator-associated infections. Clin Chest Med 2016;37:683-92.

14. Schnell D, Timsit JF, Darmon M, et al. Noninvasive mechanical ventilation in acute respiratory failure: trends in use and outcomes. Intensive Care Med 2014;40:582-91.

15. Appendini L, Patessio A, Zanaboni S, et al. Physiologic effects of positive end-expiratory pressure and mask pressure support during exacerbations of chronic obstructive pulmonary disease. Am J Respir Crit Care Med 1994;149:1069-76.

16. Slutsky AS, Ranieri VM. Ventilator-induced lung injury. N Engl J Med 2013;369:2126-36.

17. Pu XX, Wang J, Yan XB, et al. Sequential invasive-noninvasive mechanical ventilation weaning strategy for patients after tracheostomy. World J Emerg Med 2015;6:196-200.

18. Adda M, Coquet I, Darmon M, et al. Predictors of noninvasive ventilation failure in patients with hematologic malignancy and acute respiratory failure. Crit Care Med 2008;36:2766-72.

19. Lemiale V, Mokart D, Mayaux J, et al. The effects of a 2-h tria of high-flow oxygen by nasal cannula versus Venturi mask in immunocompromised patients with hypoxemic acute respiratory failure: a multicenter randomized trial. Crit Care 2015;19:380.

20. Keenan SP, Sinuff T, Burns KE, et al. Clinical practice guidelines for the use of noninvasive positive-pressure ventilation and noninvasive continuous positive airway pressure in the acute care setting. CMAJ 2011;183:E195-E214.

21. Antonelli M, Conti G, Bufi M, et al. Noninvasive ventilation for treatment of acute respiratory failure in patients undergoing solid organ transplantation: a randomized trial. JAMA 2000;283:235-41.

22. Hilbert G, Gruson D, Vargas F, et al. Noninvasive ventilation in immunosuppressed patients with pulmonary infiltrates, fever, and acute respiratory failure. N Engl J Med 2001;344:481-7.

23. Wermke M, Schiemanck S, Höffken G, et al. Respiratory failure in patients undergoing allogeneic hematopoietic SCT - a randomized trial on early non-invasive ventilation based on standard care hematology wards. Bone Marrow Transplant 2012;47:574-80.

24. Lemiale V, Mokart D, Resche-Rigon M, et al. Effect of noninvasive ventilation vs oxygen therapy on mortality among immunocompromised patients with acute respiratory failure: a randomized clinical trial. JAMA 2015;314:1711-9.

25. Azoulay E, Lemiale V. Non-invasive mechanical ventilation in hematology patients with hypoxemic acute respiratory failure: a false belief? Bone Marrow Transplant 2012;47:469-72.

26. Barbas CS, Serpa Neto A. New puzzles for the use of noninvasive ventilation for immunosuppressed patients. $J$ Thorac Dis 2016;8:E100-3.

27. Shamseer L, Moher D, Clarke M, et al. Preferred reporting items for systematic review and meta-analysis protocols (PRISMA-P) 2015: elaboration and explanation. BMJ 2015;349:g7647.

28. Parke RL, McGuinness SP, Eccleston ML. A preliminary randomized controlled trial to assess effectiveness of nasal high-flow oxygen in intensive care patients. Respir Care 2011;56:265-70.

29. Higgins JPT GS. Cochrane Handbook for Systematic Reviews of Interventions Version 5.1.0. www.cochrane-handbook.org 2011.

30. Guyatt GH, Oxman AD, Vist GE, et al. GRADE: an emerging consensus on rating quality of evidence and strength of recommendations. BMJ 2008;336:924-6.

31. Maggiore SM, Idone FA, Vaschetto R, et al. Nasal high-flow versus Venturi mask oxygen therapy after extubation. Effects on oxygenation, comfort, and clinical outcome. Am J Respir Crit Care Med 2014;190:282-8.

32. Frat JP, Thille AW, Mercat A, et al. High-flow oxygen through nasal cannula in acute hypoxemic respiratory failure. $N$ Engl $J$ Med 2015;372:2185-96.

33. Wang T, Zhang L, Luo K, et al. Noninvasive versus invasive mechanical ventilation for immunocompromised patients with acute respiratory failure: a systematic review and meta-analysis. BMC Pulm Med 2016;16:129.

34. Bienvenu AL, Traore K, Plekhanova I, et al. Pneumocystis pneumonia suspected cases in 604 non-HIV and HIV patients. Int $J$ Infect Dis 2016;46:11-17.

35. Li MC, Lee NY, Lee CC, et al. Pneumocystis jiroveci pneumonia in immunocompromised patients: delayed diagnosis and poor outcomes in non-HIV-infected individuals. J Microbiol Immunol Infect 2014:47:42-7. 